\title{
How Health Relationship Management Services (HRMS) Benefits the Elderly
}

\author{
Cynthia Meckl-Sloan
}

\author{
DynoSense Academy, CA, USA
}

\begin{abstract}
Remote health monitoring and Health Relationship Management Services (HRMS) can provide health care solutions for the elderly, the fastest-growing segment of the U.S. population. The year 2030 Problem questions whether enough resources and an operative service system will be available fourteen years from now when the elderly population will be greater than what it is today. One solution for reducing elder health care costs is home care, which is a preferable alternative to institutionalization. Many elderly have access to health services or outreach medical care, but do not use them due to lack of accessibility to safe transportation. The elderly often have problems with medication misuse stemming from the aging process, such as loss of memory, poor vision, and fixed-incomes. Seniors have dietary problems that weaken immune systems, leading to dehydration and other health issues. They also experience depression and loneliness from living alone or even with family members. The elderly who experience these problems can benefit from Health Relationship Management Services (HRMS), a new healthcare paradigm using remote health monitoring in the home.(Int J Biomed. 2016;6(2):138-142.).
\end{abstract}

Key Words: remote health monitoring • health relationship management services • elder care • family care • 2030 problem

The initial wave of Baby Boomers turned 65 in 2010, making the elderly the fastest-growing segment of the U.S. population. ${ }^{[1]}$ A key public policy concern in the field of long-term care is the impending burden an aging society will impact the care-giving system and public finances. ${ }^{[2]}$ This is called the year 2030 problem, which questions whether enough resources and an operative service system will be available in 2030, fourteen years from now when the elderly population is greater than what it is today ${ }^{[2]}$ This growth will be stimulated by Baby Boomers, who in the year " 2030 will be aged 66 to 84 - the 'young old' - and will number 61 million people. In addition to the Baby Boomers, those born prior to 1946 - the 'oldest old' - will number 9 million people in 2030." [2] This population has a higher risk of dependency stemming from chronic conditions and will use up a disparate allocation of health care resources and dollars, both public and private. ${ }^{[3]}$ A recent study shows that the economic burden of elderly care by the year 2030 will require making changes now, before the day arrives when Baby Boomers start needing long-term care. ${ }^{[2]}$ This involves taking advantage of medical and behavioral health advances to allow seniors to remain as active and healthy as possible, and to change the cultural view

Corresponding author: Cynthia Meckl-Sloan. DynoSense Academy, CA, USA.E-mail: bellacartolina@hotmail.com of growing old to ensure that all ages integrate into community life. ${ }^{[2]}$ One solution to these concerns is a new paradigm in health care called Health Relationship Management Services $(\mathrm{HRMS})^{[4]}$ as it addresses problems associated with aging, living alone, and elder health risks, such as diet, dehydration, and medication misuse.

In the past decade, there have been many efforts to reduce health spending, yet the costs of health care continue to escalate ${ }^{[3]}$ Medical innovations, such as cancer vaccines and prevention of Alzheimer's disease, may result in better health and longer life, ${ }^{[1]}$ due to new medications and innovative surgical procedures that allow people to live longer. However, with age, many new health issues have arisen, such as the combination of physical symptoms, poor eyesight, minor safety hazards, medication management issues, social isolation, forgetting appointments, mental health, and poor nutrition or malnutrition, which can be alleviated by a new healthcare paradigm called Health Relationship Management Services (HRMS) ${ }^{[5]}{ }^{[4]}$ Coronary heart disease is the most prevalent condition that affects individuals 65 and older, followed by stroke, cancer, pneumonia, and the flu. ${ }^{[5]}$ Many seniors cope with heart conditions, such as coronary artery disease, high blood pressure, atherosclerosis, vascular disease, irregular heart rhythm, hypertension and congestive heart failure, as well as breathing problems and diabetes. ${ }^{[5][6]}$ Elders 
also are prone to weakened immune systems, which leaves them open to opportunistic bacteria, viruses and diseases..$^{[5]}$ Other physiological concerns include fever, water retention, swelling of legs, diabetic conditions, dehydration, diet, and improper nutrition. ${ }^{[7]}$ Maintenance of physiological health among the aging is important, not only for the individual, but also from reducing burdens on medical services. ${ }^{[7]}$

Achieving and maintaining good nutrition is essential for elderly health and quality of life. ${ }^{[8]}$ At the root of dietary intake is availability, preparation and consumption of the proper quality and amount of foods, which may be impacted by several factors, to include social isolation and depression ${ }^{[8]}$. Imbalances of necessary nutrients and energy gained from food increases the vulnerability of the elderly to unfavorable health outcomes, such as decreasing energy levels and chronic health complications, such as type 2 diabetes, high blood pressure, heart disease, stroke, and osteoporosis. ${ }^{[9]}$ Malnutrition often goes undiagnosed due to diet and inconsistent caloric, energy, taste and access needs. ${ }^{[9]}$ Diet imbalances also can lead to lengthier hospital stays and possibility of readmission, physical, and cognitive function impairment. ${ }^{[8]}$

Seniors sometimes have difficulty recognizing bitter and salty tastes, which result in an increase in salt intake in the diet, which can lead to high blood pressure. ${ }^{[9]}$ Also, many elderly retain their capacity to appreciate sweet tastes, thus consume too many sugary snacks, beverages or desserts, which cause weight gain due to a slowing of metabolisms. ${ }^{[9]}$ Nutrition and reduced metabolisms are directly related to both weight loss and weight gain in the elderly. ${ }^{[9]}$ Moreover, the elderly, especially those suffering from chronic conditions, sometimes have less energy. ${ }^{[9]}$ Thus, reduced activity can lead to obesity ${ }^{[9]}$ Conversely, limited food access, a decrease in appetite, side-effects from medication, in addition to medical problems can create weight loss. ${ }^{[9]}$ Also, seniors tend to not purchase the foods that are rich in vitamins and minerals, due to fixed incomes or outright poverty. ${ }^{[9]}$

A common problem among the elderly is dehydration because: (1) they have a limited capacity to conserve water, (2) are not aware of their thirst, and (3) often avoid drinking fluids due to overactive bladder problems. ${ }^{[9]}$ Furthermore, the elderly are more apt to lack proper hydration during illness and in warm-weather months. ${ }^{[9]}$ Dehydration is also caused by medications and chronic medical conditions. ${ }^{[9]}$ Mild dehydration symptoms include constipation, headache, dizziness, low blood pressure, rapid heartbeat, and loss of consciousness, while more severe complications include seizures, kidney failure, swelling of the brain, heat injury, and death. ${ }^{[9]}$

Almost $90 \%$ of individuals over the age of 65 wish to live at home for as long as they can because staying in familiar surroundings offers emotional well-being. ${ }^{[10]}$ However, studies indicate that a high number of elderly who really ought to be in an assisted living care facility still live at home, and often live alone. ${ }^{[10]}$ No matter the severity of a disability or dependence, elders are encouraged to be independent at home rather than being institutionalized. There are problems associated with such an arrangement aside from health problems, among which are medication, transportation issues, depression, and loneliness. For the elderly who live at home, medication can either be a health lifeline, or it can be a calamity waiting to happen. ${ }^{[11]}$ Problems with medication are very common, and deadly results can occur if too much medication is taken or if doses are missed. ${ }^{[11]}$ There are many reasons why the elderly do not take their medications properly. Misuse of pills often occurs with seniors who have vision problems and cannot read small print on labels. ${ }^{[1]}$ Memory loss for elderly suffering from Alzheimer's disease or dementia may forget to take their medication or cause an overdose when they cannot remember whether they took their medication already. Also, seniors on fixed incomes, or are low-income, may cut back on the prescribed dose by cutting their pills in half or simply take no medication for long periods of time ${ }^{[11]}$ Elderly persons with hearing loss may not be able to hear the doctor or pharmacist's instructions. ${ }^{[11]}$ For seniors who have trouble swallowing a capsule or tablet, they tend to crush or break them, or mix the medication in with their food, but some longacting medications may be released too fast, or won't work as intended and could cause sickness. ${ }^{[1]}$

Most elderly individuals prefer self-sufficiency and would prefer to depend on their family as principal caretakers only in times of need which can take place in the home by family or caregivers. ${ }^{[12]}$ The family is, and has been, the primary means of elderly care and assistance in which elders are allowed to live at home in familiar surroundings. Family support is one social network that positively affects an elderly person's self-image, sense of importance, and feelings of being loved and respected. Home-care services provide quality of life services, such as personal care, housework assistance and help with daily tasks. Whether home care or day care, such services contribute to a sense of security and well-being, and prevent feelings of social detachment. ${ }^{[13]}$ These alternatives to institutionalizing the elderly contribute to better physical and mental capacities since elders can participate in decisionmaking, maintain their home life, have a sense of control over their lives, and establish social ties. ${ }^{[13]}$

Not all elderly, however, are fortunate enough to have family or day care. Some elderly persons have a negative day-to-day existence due to lack of income, poor health, lack of accessible and safe transportation, fear of being a crime victim, loneliness, social isolation, and limited family contact. $^{[1]]}$ Although they are eligible for health services and outreach medical care programs, many elderly people do not use them due to a lack of knowledge about how to access these services and restricted mobility because they cannot reciprocate transportation favors provided by friends or families. Community-based transportation services for seniors often require advance approval and arrangements. ${ }^{[11,14-}$ ${ }^{17]}$ Urban medical care is often avoided due to lack of safe accessible public transportation. ${ }^{[1]]}{ }^{[18]}$ Many elderly people are justifiably fearful of becoming crime victims while using public transportation. ${ }^{[11,19,20]}$

A recent Dutch study suggests that loneliness is a risk factor for the dementia. ${ }^{[21]}$ The study's findings suggest that feelings of loneliness contribute independently to the risk of dementia in later life. Individuals who feel lonely in old age are much more prone to develop dementia than those who do 
not feel lonely. People suffering from loneliness have a $64 \%$ greater risk of dementia, but this risk is dependent upon having those feelings and not simply because someone lives alone or is socially isolated, unmarried, or without social support. ${ }^{[21]}$ Additional risks for the elderly are Alzheimer's disease and other dementias that may be caused by other conditions that include depression and mild cognitive impairment.

\section{Solutions}

Homecare telemedicine for geriatric disease management is being used for many medical illnesses, such as chronic conditions and heart disease. Home telemonitoring of elderly patients with congestive heart failure confirmed a reduction of the rehospitalization rate and an improved monitoring of drug consumption by the patients. ${ }^{[22]}$ Makers of remote monitoring technology solutions for senior care empower providers to proactively provide the utmost quality care possible by gathering critical health information. Hospitals, doctor's offices, home healthcare facilities, and nursing homes are embracing wireless remote monitoring devices to communicate patient data. ${ }^{[23]}$ When equipped with the right information at the right time, caregivers now can reduce costs and increase senior independence. ${ }^{[24]}$

Telemedicine technologies can help seniors face challenges linked to social connectivity, emotional health and cognitive and physical ability. ${ }^{[25]}$ Since many seniors want to live in their homes for as long as they can, technology, such as prosthetics, wearable sensors, and other tools for daily living make it feasible. ${ }^{[25]}$ New technology is making it a possibility for adult children to monitor the activities and routines of their aging parents every day, making it possible for seniors to live in their homes rather than being transferred into an assisted living facility. ${ }^{[25]}$

The risk of seniors of being socially isolated or lonely is increasing, possibly due to living alone, retirement, poor health, and death of friends and family members. ${ }^{[26]}$ Research shows that remote monitoring shows promise for geriatric depression, and has become a key factor in helping the elderly and chronically ill live independently and safely in their homes while reducing heath care costs. ${ }^{[24]}$ Many of the problems associated with long-term elder care now and in the future can be addressed by a new healthcare paradigm called Health Relationship Management Services (HRMS). ${ }^{[4]}$

\section{Health Relationship Management Services (HRMS) Solutions}

Health Relationship Management Services (HRMS) as a solution allows the elderly to self-monitor in the safety of their own homes, thus diminishing doctor's visits, which will eliminate fears of reliance on public transportation, becoming crime victims at bus stops, or being injured while entering or exiting their own homes. Medication misuse that often leads to severe consequences can be monitored remotely, allowing advocates to step in and correct it. Essential nutrient imbalances gained from food that increases the elderly vulnerability to undesirable health outcomes can be minimized, since diet, dehydration, nutrition and dietary intake can be tracked and intervention can take place if problems arise. Seniors' independence and self-sufficiency increases, since they have empowerment over their own healthcare.

HRMS supports the diverse and changing needs of the elderly by empowering them to take control of their own health. ${ }^{[4,27]}$ HRMS can lead to lower medical costs for hospital and doctor's visits, allowing the elderly to remain independent in their own homes. An online patient portal app connects patients to the outside world to minimize isolation and loneliness. HRMS is designed to monitor an individual's health daily to detect changes in health data that would require immediate attention. New remote health care monitoring technology and HRMS is a solution to these rising health care costs, transportation worries, isolation, and barriers to health care access. ${ }^{[4]}$ HRMS can help allay those feelings by providing elderly individuals with a sense of belonging to a supportive health community in which someone is watching over them every minute of every day and monitoring their health. $\cdot^{[4]}$

\section{Case Study: Walter}

Walter is an 85-year-old diabetic widower with a history of heart disease who is being discharged from the hospital after treatment for pneumonia. He is being sent home without the benefit of a full-time caregiver since his family lives out of state. Walter has been taking four prescription medications during his hospital stay and has been prescribed two new medications at discharge to take when he gets home. His primary care provider has scheduled a follow-up appointment for two weeks after discharge. Although a hired caregiver will stop in each day for a few hours to prepare his meals, administer medication, and assist with bathing, Walter will be essentially alone once he goes home. Even though Walter still has his driver's license, his sight is failing and he doesn't feel confident to drive his car more than a few blocks to the store for groceries. Thus, he has no reliable transportation to get to his doctor's appointments across town, except for his neighbor Mrs. Conner who has offered to take him. ${ }^{[28]}$

At the hospital, Walter gets his discharge papers from a nurse, which he has trouble reading due to failing eyesight in spite of having eyeglasses. He is taken down to the hospital pharmacy to pick up his prescription medication. His neighbor, Mrs. Connor, has arranged to meet him there and then drive him home. At the pharmacy, Walter is given the remote Health Relationship Management System (HRMS) health monitoring system that his physician prescribed for him. The pharmacist explains to both Walter and Mrs. Connor how the system works, so they both clearly understand the procedure. He also provides Walter with his other prescription medications along with a lengthy set of instructions for their use. Due to his age, Walter feels overwhelmed by the complex instructions for his medication use, as there are so many pills that must be taken at different times of the day.

With the help of Mrs. Conner, Walter arrives home and gets settled in. Before she leaves, Mrs. Connor helps Walter set up his HRMS monitoring system by creating a profile on the 
downloaded app on Walter's smartphone and logs in according to the enclosed directions. At this point, Walter is prepared to use the health monitoring system every morning and evening, so it can read his personal health data. Mrs. Conner tells Walter that she will visit him whenever she can, and leaves her phone number for Walter to call if there is anything he needs or if there is an emergency. More importantly, in Mrs. Connor's absence, the HRMS system will monitor Walter's medication to prevent errors and provide him with an online connection to a caring health community.

Soon after Mrs. Connor leaves, Walter starts flicking through the health app screens on his phone, and notices that there is an interactive online magazine that looks interesting. He reads stories of people who have experienced health issues and becomes engaged in them by interacting with the authors. There are health inspired games that he likes, too, that offer points as a reward that can be cashed in for gifts. He also has an interest in health matters and enjoys reading short paragraphs about various health conditions on the app to get up-to-date information on new procedures, symptoms, or health risks.

All is well with Walter for the next few days, but since his real-time health data has been captured daily using HRMS, sudden inactivity with the system has been detected. HRMS tracks medication and has an escalation path through the system that indicates when immediate action must be taken. ${ }^{[29]}$ The Communication Center advocate calls to check in with Walter to take appropriate action. There is no answer, so the advocate calls Mrs. Connor and asks her to go visit Walter right away. She finds that he has not taken his medication because he misplaced his glasses and could not read the labels on the pill bottles. He is at risk for recurring pneumonia, but fortunately, because Mrs. Conner has been advised to visit Walter, he receives the proper attention, takes his medication, and avoids a trip to the ER for readmission to the hospital.

A few months have passed, and due to his age, Walter's children have gotten his driver's license suspended because of his failing eyesight, so he can no longer drive. Up until now, Walter actually liked living alone independently and has not felt lonely because he relished his independence, as being alone and feeling lonely are not the same. ${ }^{[30]}$ Now without the license to drive, the lack of independence and freedom has intensified Walter's sense of isolation, and he aches for social contact. Feeling lonely with the perceived absence of social attachments increases the risk of cognitive decline and is associated with the onset of dementia. With HRMS, Walter's contact to the outside world consists of watching television, speaking to some friends occasionally on the phone, and visits from Mrs. Connor. More importantly, Walter is also connected to a caring healthcare community through HRMS that gives him the confidence that there is someone who cares about him and is watching over him, albeit remotely. By connecting to HRMS, Walter's sense of loneliness has abated, and he continues to live happily by himself independently in his own home.

With the introduction of new technology that helps seniors live safely and productively in their own homes, a story like Walter's could really happen. HRMS is a new healthcare paradigm that is designed to help seniors who are living at home, either with caregivers or by themselves to avoid some of the problems associated with aging. Remote health monitoring and HRMS will be able to provide elderly persons with independence and control over their health issues. For elderly individuals who either live alone or stay with family, HRMS will one day reduce medical visits and associated difficulties with transportation. From the online patient platform, patients' feelings of isolation and loneliness will be assuaged by the knowledge that someone is looking out for their health and that they are part of a caring health community.

\section{References}

1. Rand. Future health and medical care spending of the elderly. Implications for medicare. (2016). Retrieved from http:// www.rand.org/pubs/research_briefs/RB9146-1/index1.html

2. Knickman JR, Snell EK $\overline{\text {. The }} 2030$ problem: caring for aging baby boomers. Health Serv Res. 2002 Aug;37(4):849-84. 3. Experton B, Li Z, Branch LG, Ozminkowski RJ, MellonLacey DM. The impact of payor/provider type on health care use and expenditures among the frail elderly. Am J Public Health. 1997 Feb;87(2):210-6.

4. Tehrani N. Health Relationship Management Services (HRMS). A New Healthcare Paradigm Using the 5Rs. Int J Biomed. 2016; 6(1):87-89. doi: 10.21103/Article6(1)_CC1.

5. Levy S. The most common issues of aging. (2015). Aging Care.com. Retrieved from https://www.agingcare.com/ Articles/common-issues-of-aging-102224.html

6. Nabili S. What are the most common diseases and conditions seniors face as they age? (2015). MedicineNet. com. Retrieved from http://www.medicinenet.com/senior health/page2.html

7. Nigam Y, Knight J, Bhattacharya S, Bayer A. Physiological changes associated with aging and immobility. J Aging Res. 2012;2012:468469. doi: 10.1155/2012/468469.

8. Sharkey JR, Branch LG, Zohoori N, Giuliani C, BusbyWhitehead J, Haines PS. Inadequate nutrient intakes among homebound elderly and their correlation with individual characteristics and health-related factors. Am J Clin Nutr. 2002 Dec;76(6):1435-45.

9. Morris I. Nutrition problems in the elderly. SF Gate. (2016). Retrieved from http://healthyeating.sfgate.com/ nutrition-problems-elderly-1370.html

10. AARP. When it's time for a nursing home. (2016). Retrieved from http:/www.aarp.org/home-family/caregiving/ senior-housing/

11. Sollitto M. Top 6 Medication Problems and How to Prevent Them. (2015). AgingCare.com. Retrieved from https:/www.agingcare.com/Articles/medication-problemselderly-people-have-146111.html

12. Health care and public transportation use by poor and frail elderly people. Rittner B, Kirk AB.Soc Work. 1995 May;40(3):365-73.

13. Ron P. Self-esteem among elderly people receiving care insurance at home and at day centers for the elderly. Int Psychogeriatr. 2007 Dec;19(6):1097-109.

14. Krout JA. Senior center linkages in the community. Gerontologist. 1986 Oct;26(5):510-5.

15. Auslander GK, Litwin H. Social support networks and formal help seeking: differences between applicants to social services 
and a nonapplicant sample. J Gerontol. 1990; 45(3):S112-9.

16. Coe RM, Wolinsky FD, Miller DK, Prendergast JM. Complementary and compensatory functions in social network relationships among the elderly. Gerontologist. 1984 Aug;24(4):396-400.

17. Stoesz D, Karger HJ. Welfare reform: from illusion to reality. Soc Work. 1990 Mar;35(2):141-7.

18. Jirovec RL, Erich JA, Sanders LJ. Patterns of senior center participation among low-income urban elderly. Journal of Gerontological Social Work. 1989; 13(3-4):115-32.

19. Clark SP, Adler LL, Adler HE. Urban crime and the elderly. International Journal of Group Tensions. 1983; 1(1-4):76-83.

20. Patterson AH. Fear of crime and other barriers to use of public transportation by the elderly. Journal of Architectural and Planning Research.1985; 2:277-88.

21. Holwerda TJ, Deeg DJ, Beekman AT, van Tilburg TG, Stek ML, Jonker C, Schoevers RA. Feelings of loneliness, but not social isolation, predict dementia onset: results from the Amsterdam Study of the Elderly (AMSTEL). J Neurol Neurosurg Psychiatry. 2014 Feb;85(2):135-42. doi: 10.1136/ jnnp-2012-302755.

22. Spinsante S, Antonicelli R, Mazzanti I, Gambi E. Technological approaches to remote monitoring of elderly people in cardiology: a usability perspective. Int J Telemed Appl. 2012;2012:104561. doi: 10.1155/2012/104561.

23. Lewis M. Remote patient monitoring market to double by 2016. Information week. (2012). Retrieved from http://www. informationweek.com/mobile/remote-patient-monitoringmarket-to-double-by-2016/d/d-id/1105484

24. Fallon Health. New Study Finds that Remote Monitoring Reduces Long-Term Care Days, ER Visits and Hospital Admissions. Health IT outcomes. (2015). Retrieved from http:// www.healthitoutcomes.com/doc/new-study-finds-monitoringreduces-long-days-er-visits-and-hospital-admissions-0001?atc $\sim \mathrm{c}=771+\mathrm{s}=773+\mathrm{r}=001+\mathrm{l}=\mathrm{a} \& \mathrm{sf35521560}=1$

25. Miliard, M. Report to President Obama claims remote monitoring technologies, robust Internet are essential as Americans live longer. Healthcare IT news. (2016). Retrieved from http://www.healthcareitnews.com/news/pcast-toutstelehealth-wearable-sensors-key-keeping-aging-populationconnected

26. British Columbia Ministry of Health. Children's, Women's and Seniors Health Branch. (2004). Social isolation among seniors: An emerging issue. Retrieved from http:// www.health.gov.bc.ca/library/publications/year/2004/Social Isolation Among Seniors.pf

27. PwC Health Research Institute. (2016). Thriving in the new economy. Health industry issues of 2016. Retrieved from http://editiondigital.net/publication/?i=287438\# \{ “issue_ id":287438,"page":0\}

28. Moulds K, Epstein K. Do post-discharge telephone calls to patients reduce the rate of complications? The Hospitalist. (2008). Retrieved from http://www.the-hospitalist.org/article/ do-post-discharge-telephone-calls-to-patients-reduce-therate-of-complications/

29. Larkin M. Dr. Eric Topol: Digital healthcare will put the patient in charge. Elsevier. (2014). Retrieved from https:// www.elsevier.com/connect/Dr-Eric-Topol-Digital-healthcarewill-put-the-patient-in-charge

30. Campbell D. Dementia linked to loneliness, study finds. Dutch research reveals that people with feelings of loneliness more likely to suffer from mental deterioration. The Guardian. (2012). Retrieved from http://www.theguardian.com/ society/2012/dec/10/loneliness-dementia-link. 